\title{
PROTOTYPE ULTRASONIC WAYFINDER WITH HAPTIC FEEDBACK FOR AN IOT ENVIRONMENT
}

\author{
Soonja Yeom ${ }^{1}$, Riseul Ryu ${ }^{1}$, Eunbin Pak $^{2}$ and Pankoo Kim ${ }^{2}$ \\ ${ }^{1}$ School of Technology, Environment and Design, University of Tasmania, Sandybay, Hobart, Australia \\ ${ }^{2}$ Department of Computer Engineering, Chosun University, Gwangju, Republic of Korea
}

\begin{abstract}
Sensor technology for small mobile devices is developing quickly, prices for equipment are dropping rapidly and IoT infrastructure is increasingly accessible. These developments have made it possible to build a haptic glove with a vibrating interface to help a person with visual impairment to move around. This haptic sensing glove will guide the user as an additional device - on top of what they use for guidance at present. This article describes a prototype which is undergoing user testing and can be further tied into a smart home environment.
\end{abstract}

\section{KEYWORDS}

Sensor Technology, Haptic Feedback, Wearable Technology

\section{INTRODUCTION}

Pervasive computing and the Internet of Things (IoT) have stimulated the development of many new assistive devices. It is possible to incorporate sensors such as acoustic, inductive, capacitive, temperature, humidity, pressure, location, and many more. Haptic feedback provides a person with sensory information through the skin using vibration or force-feedback responses. Commercial organizations have moved very quickly into this design space, particularly Sunu (smart-watch), HandSight (cameras on glove), and others. Arduino and Raspberry Pi are examples of the computing platforms currently in use. Sonar or ultrasonic transducers enable the production of lighter equipment with improved functionalities. Sonar as a means of assistive navigation has been used extensively in maritime environments to detect animals (D'Amico and Pittenger, 2009, Evans and Awbrey, 1988). As an assistive technology, there are projects for the blind which upgrade their walking sticks with an ultrasonic sensor (Amemiya and Sugiyama, 2010).

Similar projects have been undertaken worldwide and most devices can only provide one or two designated functions. The size of the completed device is small enough to embed on a shoe, a walking stick, or on a wheelchair. A sonar sensor can detect something less than a meter from an individual user. This study uses a glove to attach a sonar sensor on a Raspberry Pi 0, whereas the Tacit glove (Hoefer, 2011) carries two sonar sensors with an Arduino controller actuating vibrating motors on a glove.

\section{BACKGROUND}

The Raspberry Pi is a small, powerful, cheap, hackable and education-oriented computer board introduced in 2012 (Vujović and Maksimović, 2014, Nikhade, 2015). It has the capacity to build a wireless sensor network (WSN), with a sensing unit, processor, storage, and transceiver.

The project was to design and build a device worn on a single hand by a visually impaired person. Each wearable glove carries a sonar sensor and a set of haptic feedback devices, connected to a Raspberry Pi Zero (RPi0). As the RPi0 has minimal mass, size, and power which combines with a minimal mass and size $5 \mathrm{~V}$ power pack. One of the limitations of the Raspberry Pi for this kind of application is its relatively large power consumption (Vujović et al., 2014). The glove would provide haptic feedback to the wrist/hand of the wearer, via vibration, on the relative positional-distance of objects. The positional-distance would be 
calculated through data provided by sonar sensors contained on the glove. The sonar sensors should have a documented range of up to 4 metres, however they worked up to a distance of 1.5 meters in the test. Some of the contributing factors include distance, angle, signal absorbency, and power input (Borenstein and Koren, 1988).

The RPi0 would operate headless - that is, without any screen or keyboard which reduces the need for peripheral components. The aim is to provide:

(a) A wearable platform that can be used by sight-impaired persons to sense their immediate environment by hand-waving.

(b) A device which can be modified by software to provide a wider range of functions compared to other similar devices.

A similar haptic handheld wayfinder for people with visual impairment was reported in 2010 (Amemiya and Sugiyama, 2010). This was positively evaluated by people with visual impairments. It needed extra devices and a miniaturized force display. Adding the device to a glove produced a similar project, Tacit Glove, which was tested in 2011 (Hoefer, 2011). Another approach is putting the device on cane. The Raspberry Pi was compared with other wireless sensor nodes such as MicaZ, TelosB, Iris, Cricket, and Lotus in terms of size, cost, power, memory, and flexibility (Vujović \& Maksimović, 2014). The Raspberry Pi has better processing power, memory, connectivity, and multipurpose usage (USB) with a cheaper cost. However, power consumption was the area identified for improvement. In the prototype, Python 2 was used for programming instead of Python 3, since some libraries have not yet been ported to Python 3.

\section{PROTOTYPE}

\subsection{What it does?}

The Raspberry Pi 3 (RPi3) is the fully functional current model that is widely available. The Raspberry Pi Zero (RPi0) is lighter than the RPi3 as well as less power demanding. In the project, the RPi0 is used with an ultrasonic sensor powered by an auxiliary $5 \mathrm{~V}$ battery pack. It also supports a WIFI dongle for connectivity in headless configuration. For haptic feedback, a micro servomotor, 'SG90' was tested, followed by a vibration module, with the thought that vibration module would be an easier option to use.

The board was modified as shown in the diagram below (Figure 1) and connected to the ultrasonic sensor via the 5V General Purpose Input/Output (GPIO) pin. The 5V pin of the ultrasonic sensor is connected to GPIO Pin 2 (which is for $5 \mathrm{~V}$ power) on the RPi0. The trigger signal is connected to Pin 16 (GPIO23), the Echo line is connected to Pin18 (GPIO24) and Ground to Pin6 (Ground).

A USB hub board (Figure 2) was attached to the Raspberry Pi so that the WIFI dongle could be attached to the top of the RPi0, rather than protruding from a side (where it is easily brokened or damaged).
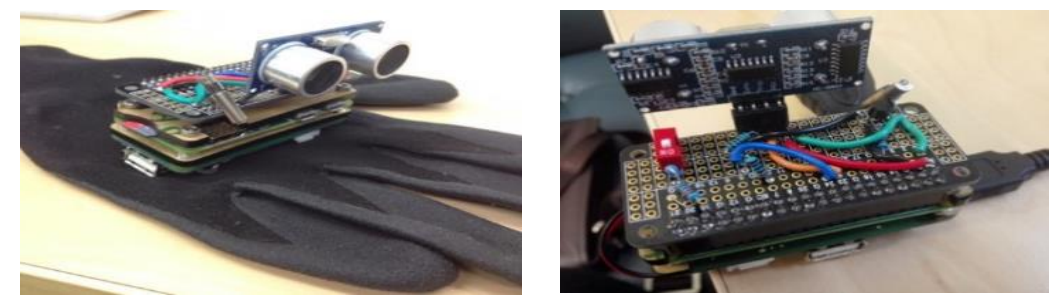

Figure 1. Assembled version before putting it on glove

The prototype uses the ultrasonic sensor for object recognition, while the existing systems in this field commonly use the fixed cameras (Wang et al., 2017, Yi et al., 2013) or radio frequency (Dionisi et al., 2012). The ultrasonic sensor was successfully assembled on the RPi0 by using the breadboard and jumper cable through an expanded GPIO board (BreakHAT). A switch and a vibrator were placed on the board and the circuitary test was performed.. A way is currently being sought to add the servomotor or vibrator or LCD screen to the glove. But the LCD screen was found to be too large and impractical for use, so the project 
switched to a virtualised screen through VNC viewer. It should be noted that the virtualised screen is only for developers as an auxiliary visual aid for use when testing. More experiments are needed to determine whether RPi0 can provide enough power for two different sensors. The ultrasonic sensor needs a pair of resistors to lower the input $5 \mathrm{~V}$ voltage to $3.3 \mathrm{~V}$.

All the devices, including ultrasonic sensors and High-Definition Multimedia Interface (HDMI) will be connected to a small LCD display and battery pack when completed, excluding keyboard, mouse and WIFI dongle. Power was supplied with a 5V battery kit with USB charging input and a switch attached (Figure 3). If running more than just the WIFI dongle the battery pack will need to be upgraded - which could be a powerbank or any external portable battery suitable for a mobile phone. The input should be about $1 \mathrm{~A}$ to source enough power to the RPi0.

In the experiment for measuring distance, a WIFI dongle with a battery pack was used due to a need for lesser than $5 \mathrm{~V}$ supplied from the $5 \mathrm{~V}$ battery. It was controlled via Secure Shell ( $\mathrm{SSH}$ ) through the phone hotspot WIFI and the Android application 'connectbot'. The ultrasonic sensor works well and updates the distance reading as it changes. The vibrator or servomotor produces haptic feedback when the distance is closer than $30 \mathrm{~cm}$ or $100 \mathrm{~cm}$. Detecting an obstacle at $30 \mathrm{~cm}$ may be too short to avoid when the user is walking, hence different signals can be given to the user at 1 meter and again at $50 \mathrm{~cm}$ with a longer and stronger vibration. The simple on/off switch is attached to the prototype so that a user who is visually impaired can turn the prototype on and off without entering the commands on the device through a keyboard. It needs further work to implement a mobile application so that the user can issue voice commands to connect to the device, run the system, and turn the device off.

Due to power concerns, it is proposed that the prototype uses a bigger battery such as a small mobile phone battery or dedicated battery pack to replace current small $5 \mathrm{~V}$ battery for further development work. It will support the vibrator and ultrasonic sensor as well as the extra USB hub board for the WIFI dongle.

\subsection{Difficulties}

One of the major difficulties is the power consumption of the device (Vujović \& Maksimović, 2014). This may be addressed in future through programming to reduce clock cycles when not in motion, or by choosing lower power consumption sensors and actuators.

Further testing by the Tacit Project looked at various implementations of haptics on devices like headbands and vests and highlighted several problems related to cost (vest) and object height (headband) (Hoefer, 2011). While the Tacit Project finally settled on a wrist and glove mounted device, of which this project shares a similar design, some problems were identified with range of movement that need to be addressed in future research.

One such issue relates to the physical action of 'searching' using a wide straight-arm motion which can produce variable results between 80 and 120 degrees. A bent arm at the elbow can account for a greater range of motion but is still blocked by the body and range of motion issues that may be present. Further physical issues with the device and range of motion come from the height of the device in the sweep. Which may exclude objects that are nearby but out of the cone. Furthermore if the sensor is pointed near the ground then objects that are further up may be excluded. Simple testing resulted in the use of a figure-eight like motion to scan distant and near objects, but prolonged use will result in discomfort.

Another broader problem comes from the use of ultrasonic sensors which have technical difficulties that present problems in this style of device, like smoothness of surface, softness of surface, and size of the object (Borenstein and Koren, 1988). A solution for this is to use the properties of RPi0 to run algorithms to increase the effectiveness of the ultrasonic sensors, with the possible inclusion of different sensor types to counteract the negative traits.

\section{CONCLUSION}

The purpose of this project is to create a prototype device that uses ultrasonic sensors in a lightweight environment to provide haptic feedback based on environmental cues. This was achieved through using an RPi0 mounted on a glove, which has the benefit of being extensible through software and added to smart home 
environments. Extending on the features of the device in a smart home environment will be the focus for further research after the functionality of the device has been tested.

The vibration gives feedback to the users in terms of the distance from an object; however, this design has yet to be validated in the project group. Full user testing will evaluate the system's feasibility which will require social science ethics approval. This will require permission to undertake usability trials for a community group with visual difficulties. User testing will be conducted to evaluate the accuracy of the system by comparing the real distance and the distance of feedback vibration. The prototype needs a glove or bracelet to put on (Figure 1). The prototype also needs to be used in conjunction with a cane, as it is currently unable to detect obstacles under knee level.

Because the platform can be extended by software, it can arrange to collect data about personal mobility and safety. The infrastructure can be expanded to provide an alarm when the person is assumed in trouble, by identifying an abnormal pattern of movement. The alarm can also be issued to a pre-arranged destination (remote receiver). This extension of the project can be beneficial to the user and caretaker but will require additional ethical approvals. Security and privacy are other important issues to be considered as the project moves forward.

Microcontrollers (MCUs) have a purpose-driven, low-cost nature. Wearable technology combined with a cloud-based wellness program enables individuals to self monitor manage and share their data with healthcare providers and others. By leveraging the properties of IoT and smart homes, the device can be used to build up an environmental pattern of object layout within the home. By using a central storage device to communicate with the RPi0, it is possible to map the environment, resulting in a finely-tuned system with location awareness.

\section{ACKNOWLEDGEMENT}

This research was supported by the Korean MSIT (Ministry of Science and ICT), under the National Program for Excellence in SW , supervised by the IITP (Institute for Information \& communications Technology Planning\&Evaluation)(2017-0-00137).

\section{REFERENCES}

amemiya, T. \& Sugiyama, H. 2010. Orienting kinesthetically: A haptic handheld wayfinder for people with visual impairments. ACM Transactions on Accessible Computing (TACCESS), 3, 6.

Borenstein, J. \& Koren, Y. 1988. Obstacle avoidance with ultrasonic sensors. IEEE Journal on Robotics and Automation, 4, 213-218.

D'amico, A. \& Pittenger, R. 2009. A brief history of active sonar. Space and Naval Warfare Systems Center San Diego $\mathrm{Ca}$.

Dionisi, A., Sardini, E. \& Serpelloni, M. Wearable object detection system for the blind. 2012 IEEE International Instrumentation and Measurement Technology Conference Proceedings, 13-16 May 2012 2012. 1255-1258.

Evans, W. E. \& Awbrey, F. T. 1988. Natural history aspects of marine mammal echolocation: feeding strategies and habitat. Animal Sonar. Springer.

Nikhade, S. G. Wireless sensor network system using Raspberry Pi and zigbee for environmental monitoring applications. Smart Technologies and Management for Computing, Communication, Controls, Energy and Materials (ICSTM), 2015 International Conference on, 2015. IEEE, 376-381.

Vujović, V. \& Maksimović, M. Raspberry Pi as a wireless sensor node: performances and constraints. Information and Communication Technology, Electronics and Microelectronics (MIPRO), 2014 37th International Convention on, 2014. IEEE, 1013-1018.

Wang, H., Katzschmann, R. K., Teng, S., Araki, B., Giarré, L. \& Rus, D. Enabling independent navigation for visually impaired people through a wearable vision-based feedback system. 2017 IEEE International Conference on Robotics and Automation (ICRA), 29 May-3 June 2017 2017. 6533-6540.

Yi, C., Flores, R. W., Chincha, R. \& Tian, Y. 2013. Finding Objects for Assisting Blind People. Network modeling and analysis in health informatics and bioinformatics, 2, 71-79. 